Tratamiento del cáncer de próstata en función de la esperanza de vida, la comorbilidad y las guías de práctica clínica

\title{
Treatment of prostate cancer according to life expectancy, comorbidity and clinical practice guidelines
}

\author{
J.L. Pérez-Gracia, M.E. Rodríguez Ruiz, J.P. Fusco, A. Gúrpide
}

\section{Sr. Director:}

En un número anterior de la revista de Anales del Sistema Sanitario de Navarra, Barceló y col realizaron una interesante y útil revisión de los pacientes con cáncer de próstata tratados en un gran centro hospitalario español durante un año, centrándose en sus características basales, el tratamiento realizado y el grado de seguimiento de las Guías de Práctica Clínica (GPC) y las complicaciones asociadas a los tratamientos realizados ${ }^{1}$.

La serie de casos recogidos es suficientemente amplia (114 pacientes) y los resultados son relevantes y merecen un valoración profunda. La mayoría de los pacientes presentaban buen pronóstico (el 92\% tenia una esperanza de vida mayor de 10 años) y el $86 \%$ presentaban comorbilidad media o baja, según el índice de Charlson ${ }^{2}$.

Significativamente, en el $38,6 \%$ de los casos no se siguieron las recomendaciones de las GPC utilizadas como referencia en el estudio (Guías de Cáncer de próstata de la Sociedad Europea de Urología del año $2011^{3}$ que corresponde al año en el cual se recogieron los casos del trabajo), y este porcentaje llegó al $58,1 \%$ en los casos de riesgo alto. Aunque las GPC utilizadas no son de obligado cumplimiento, están avaladas por una prestigiosa sociedad médica. Estos resultados concuerdan con los de otros estudios similares ${ }^{4,5}$. Por tanto el alto porcentaje de falta de seguimiento de las mismas debe invitar a una reflexión sobre el grado de cumplimiento de las recomendaciones basadas en la evidencia y también sobre el éxito conseguido en la difusión y aplicación de las diferentes GPC.

La toxicidad observada reflejó los eventos esperados tras los tratamientos habitualmente empleados en el cáncer de próstata $^{6-8}$, sin embargo, el alto porcentaje de casos de incontinencia (70\%), disfunción eréctil (48\%) y enfermedades cardiovasculares tras hormonoterapia (17\%) revelan que a pesar de las grandes mejorías que se han producido en el tratamiento del cáncer de próstata, es preciso desarrollar nuevas estrategias que mejoren la calidad de vida de los pacientes. En este sentido, la introducción del tratamiento con docetaxel en el momento de iniciar el bloqueo hormonal conlleva una importante mejoría de la supervivencia en pacientes con enfermedad avanzada hormonosensible 9 . Por tanto, serán necesarios estudios similares a éste, que valoren el grado de incorporación de
Departamento de Oncología. Clinica Universidad de Navarra. Pamplona. Navarra.

\author{
Correspondencia: \\ Jose Luis Pérez Gracia \\ Departamento de Oncología \\ Clínica Universidad de Navarra \\ 31008 Pamplona \\ E-mail: jlgracia@unav.es
}


esta nueva estrategia a la práctica asistencial habitual.

La posibilidad de extrapolar los resultados y sus conclusiones a otros centros hospitalarios similares es discutible, dado que pueden existir diferencias significativas en distintas unidades asistenciales. No obstante, sí cabe afirmar que cuando menos, estos resultados apoyan la realización de estos procesos de valoración en otros centros similares, a nivel nacional y la adopción de medidas de mejora en función de los resultados observados.

\section{BIBLIOGRAFÍA}

1. Barceló Obrador A, Ramos M, de la Iglesia M, ZAFoRTEZA M. Tratamiento del cáncer de próstata en función de la esperanza de vida, la comorbilidad y las guías de práctica clínica. An Sist Sanit Navar 2014; 37: 339-348.

2. Charlson ME, Pompei P, Ales KL, MacKenzie CR. A new method of classifying prognostic comorbidity in longitudinal studies: development and validation. J Chronic Dis 1987; 40: 373-383.

3. Heidenreich A, Bellmunt J, Bolla M et al. Eau guidelines on prostate cancer. Part 1: screening, diagnosis, and treatment of clinically localised disease. Eur Urol 2011; 59: 61-71.

4. Morgia G, Russo GI, Tubaro A et al. Patterns of prescription and adherence to European As- sociation of Urology guidelines on androgen deprivation therapy in prostate cancer: an Italian multicentre cross-sectional analysis from the Choosing Treatment for Prostate Cancer (CHOICE) study. BJU Int 2015; agosto31.doi 10.1111/bju.13307.

5. Kuykendal AR, Hendrix LH, Salloum RG, Godley PA, CHEN RC. Guideline-discordant androgen deprivation therapy in localized prostate cancer: patterns of use in the medicare population and cost implications. Ann Oncol 2012; 24: 1338-1343.

6. Hunter GK, Reddy CA, Klein EA, Kupelian P, ANGermeier K, UlchaKer $\mathrm{J}$ et al. Long-Term (10-Year) gastrointestinal and genitourinary toxicity after treatment with external beam radiotherapy, radical prostatectomy, or brachytherapy for prostate cancer. Prostate Cancer 2012; 2012. DOI: 10.1155/2012/853487.

7. Xiong T, Turner RM, Wei Y, Neal DE, Lyratzopoulos G, Higgins JPT. Comparative efficacy and safety of treatments for localised prostate cancer: an application of network meta-analysis. BMJ Open 2014 May15; 4 (5): e004285.doi 10.1136

8. Resnick MJ, Koyama T, Fan KH, Albertsen Pc, Goodman M, Hamilton as et al. Long-term functional outcomes after treatment for localized prostate cancer. N Engl J Med 2013; 368: 436-445.

9. Sweeney CJ, Chen Y-H, Carducci M, Lin G, Jarrard DF, EISENBERGER M et al. Chemohormonal therapy in metastatic hormone-sensitive prostate cancer. N Engl J Med 2015; 373: 737-746. 